\title{
The research determines appropriate parameters in the synthesis process of syringic acid grafted chitooligosaccharides
}

\author{
Bui Van Hoai ${ }^{1,2}$, Dai-Nghiep Ngo ${ }^{1,3, *}$
}

${ }^{1}$ University of Science, Biochemistry, Faculty of Biology-Biotechnology, Vietnam National University Ho Chi Minh City (VNU-HCM)

${ }^{2}$ Center of Experiment and Practice, Ho Chi Minh City University of Food Industry (HUFI)

${ }^{3}$ Lab of Enzyme Technology, University of Science, Vietnam National University Ho Chi Minh City (VNU-HCM)

\section{Correspondence}

Ngo Dai Nghiep, University of Science, Biochemistry, Faculty of Biology-Biotechnology, Vietnam National University Ho Chi Minh City (VNU-HCM)

Lab of Enzyme Technology, University of Science, Vietnam National University Ho Chi Minh City (VNU-HCM)

Email: ndnghiep@hcmus.edu.vn

\section{History}

- Received: 2018-12-21

- Accepted: 2019-08-09

- Published: 2019-08-22

DOI :

https://doi.org/10.32508/stdj.v22i3.1289

\section{Check for updates}

\section{Copyright}

(c) VNU-HCM Press. This is an openaccess article distributed under the terms of the Creative Commons Attribution 4.0 International license.

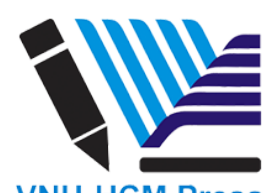

\begin{abstract}
Introduction: The aim of this study is to determine appropriate parameters in the synthesis of syringic acid onto chitooligosaccharides (COSs) with an ascorbic acid/hydrogen peroxide redox pair in order to obtain the derivative with the highest grafting degree. Methods: In this study, syringic acid grafted COSs, catalysed by an ascorbic acid/hydrogen peroxide redox pair were investigated. The synthesis conditions were investigated, including the mass ratio between syringic acid and COSs, $\mathrm{pH}$, temperature and synthesis time. Characteristics of the derivative were evaluated by Thin Layer Chromatography (TLC), Ultraviolet-Visible (UV-vis) and FourierTransform Infrared (FT-IR) spectroscopy. The activities of COSs and derivative were evaluated by antimicrobial ability. Results: The results showed, that the best conditions for the synthesis were the mass ratio between syringic acid and COSs at $0.5: 1, \mathrm{pH}$, temperature $27^{\circ} \mathrm{C}$, for 6 hours with grafting degree at $32 \%$. The TLC assay showed, that free ascorbic acid and syringic acid are not present in the product. The UV-vis and FT-IR data confirmed, that syringic acid was successfully conjugated onto COSs. Furthermore, the antibacterial assay showed that syringic acid grafted onto COSs had minimum inhibitory concentration against foodborne pathogenic bacteria at $1 \%$. Conclusion: The syringic acid onto chitooligosaccharides were successfully synthesized by free radical mediated grafting method with an ascorbic acid/hydrogen peroxide redox pair. The grafting degree of syringic acid onto COSs was greatly affected by many factors, including COSs, syringic acid, $\mathrm{pH}$, as well as temperature and time of reaction. Moreover, the new derivative showed enhanced antibacterial capabilities, as compare to free COSs.
\end{abstract}

Key words: chitooligosaccharides, FTIR, antimicrobial, syringic acid, UV-vis

\section{INTRODUCTION}

Chitin is the second most abundant polysaccharide, mainly extracted from the exoskeleton of sea creatures, such as crayfish, lobster, prawns, crab and shrimp. Besides, various microorganisms also produce chitin (for instance, cell walls of fungi and yeasts contain chitin). Chitin, in the form of films and fibers, is used in numerous applications (e.g. biosensor) ${ }^{1}$. Chitin shows various bioactivities, such as antioxidative $^{2}$, immunostimulanting ${ }^{3}$, enzyme inhibitory, antimicrobial, anticancer and anticholesteremic activities ${ }^{4}$. Chitosan, a partially deacetylated polymer of Nacetyl glucosamine, is prepared by alkaline deacetylation from chitin, with the degree of deacetylation of chitin reaching about $50 \%{ }^{1}$. Chitosan is also biologically active and possesses antioxidative ${ }^{5}$, antimicrobial $^{6,7}$, immunity enhancing ${ }^{8}$ and anticancer ${ }^{9}$ features. Due to its non-toxic, non-antigenic, biocompatible and biodegradable properties, chitosan has wide applications in food, tissue engineering, pharmaceutical, textile, agriculture, water treatment and cosmetics industries ${ }^{10}$. Although chitin and chitosan have many advantages, they have poor solubility in water and thus have limited application in the living systems ${ }^{11,12}$. Chitooligomers (COSs), partially hydrolyzed by enzyme and chemistry methods products of chitosan, are of great interest in pharmaceutical and medicinal applications due to their noncytotoxic and high water-soluble properties ${ }^{13}$. The COSs and its derivatives offer a broad spectrum of health-beneficial biological activities for humans, including anti-inflammatory, anti-oxidant, anti-cancer, anti-microbial activities ${ }^{12,14,15}$. In recent years, an interest to the synthesis of COSs derivatives to enhance its has increased.

Natural phenolic compounds from the plant source s and food products have been showed to possess bioactivities such as anti-oxidant, anti-microbial, antiinflammatory, anti-mutagenic, anti-allergenic and prevention of diabetes and cancer diseases effects ${ }^{17}$. Grafting of phenolic compounds onto COSs in order to enhance their activity is of particular interest to researchers. The syringic acid is among the m. Until now, most of COSs synthesis uses only chemical method with carbodiimide based 
<smiles>[Y]C1=C(O)C(=O)OC1C(O)CC(=O)O</smiles>

Ascorbic acid $\left(\mathrm{AscH}_{2}\right)$<smiles>CCC(O)C1OC(=O)C(O)=C1O</smiles>

$\mathrm{AscH} \cdot$

Asc:-

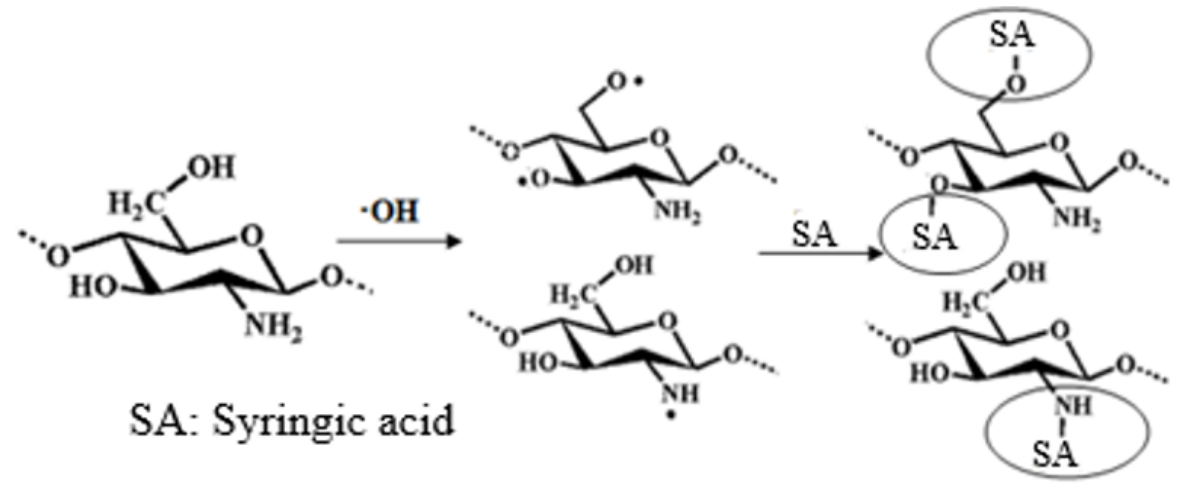

Figure 1: Proposed mechanisms for the synthesis of syringic acid-g- $\cos s^{16}$.
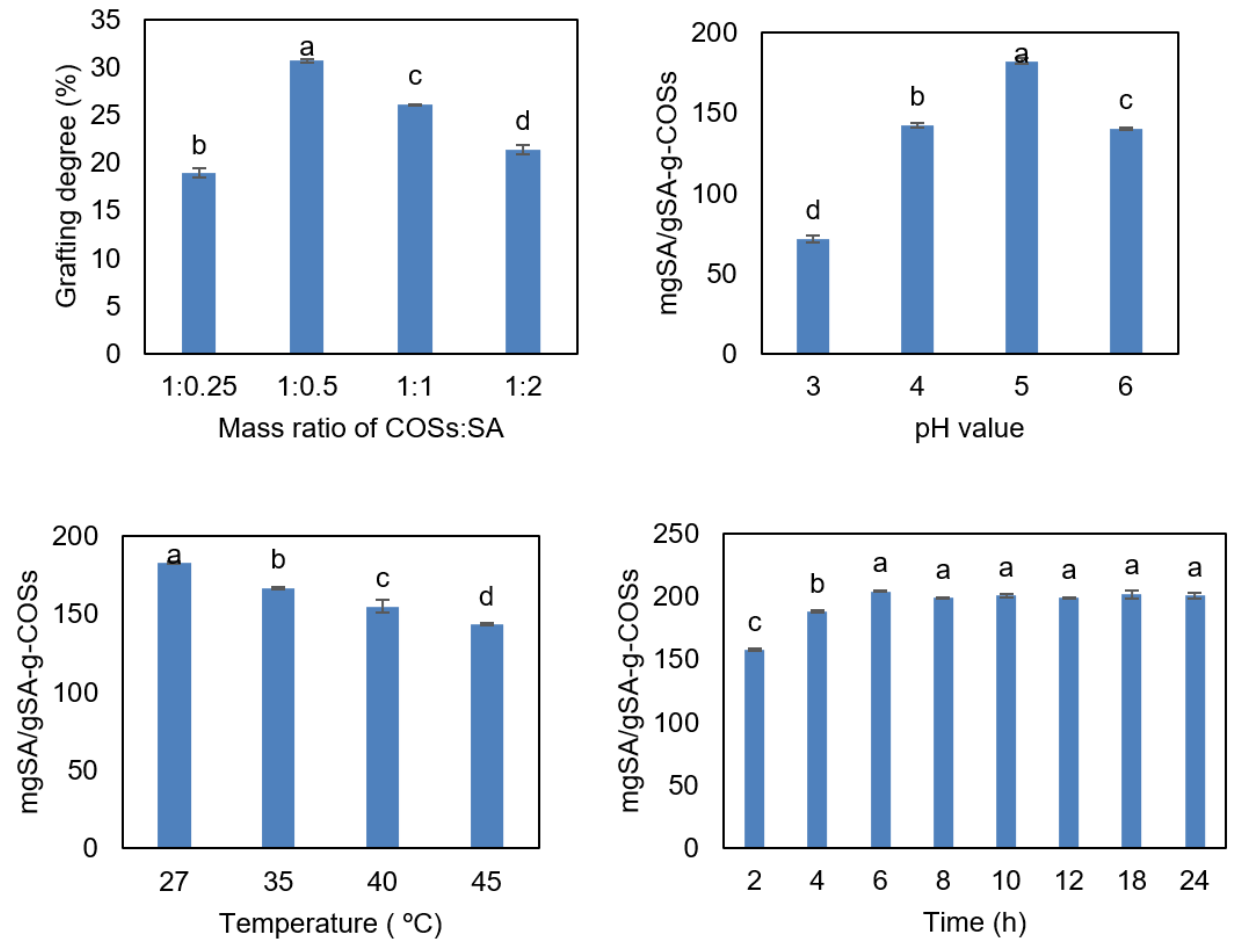

Figure 2: The results of optimization of grafting conditions: (A) Influence of COSs:SA ratio (COSs:SA from 1:0.25 to $1: 2, \mathrm{pH} 5$, at $27^{\circ} \mathrm{C}$, for 24 hours); (B) Influence of $\mathrm{pH}$ (pH ranged from 3 to $6, \mathrm{COSs}: \mathrm{SA}$ ratio was 1:0.5, at $27^{\circ} \mathrm{C}$, for 24 hours); (C) Influence of temperature (temperature from $27^{\circ} \mathrm{C}$ to $45^{\circ} \mathrm{C}, \mathrm{COSs}: \mathrm{SA}$ ratio was 1:0.5, pH 5, for 24 hours); (D) Influence of time (time from 2 hours to 24 hours, COSs:SA ratio was 1:0.5, pH 5, at $27^{\circ} \mathrm{C}$ ). All statistical analyses were performed on the data, derived from three independent experiments and the data are shown as means \pm SD. Means with different letters $(a, b, c, d)$ are significantly different at $p<0.05$ level. 
chemical coupling reagents, such as 1-ethyl-3-(3dimethylaminopropyl) carbodiimide (EDC) and Nhydroxysuccinimide (NHS) reaction, or EDC and Dicyclohexylcarbodiimide (DCC) ${ }^{12,14}$. EDC and NHS, or EDC and DCC reagents are usually required in large amounts, considered environmentally disadvantageous and difficult to remove completely from the reaction media. As compared to these approaches, free radical mediated grafting of COSs is considered to be quicker and eco-friendlier. The redox pair system does not generate toxic reaction products and it is possible to perform the reaction at room temperature, thus avoid ing degradation of antioxidants ${ }^{18}$. Efficiency of synthesis is affected by many factors, such as the mass ratio of the grafting substrate to COSs, $\mathrm{pH}$, temperature and time. Until now, the method of free radical mediated reagent, in order to graft syringic acid onto COSs is not reported yet. Thus, the aim of this work is to determine optimal condition in the synthesis process and to evaluate, whether syringic acid can be successfully grafted onto COSs, using ascorbic acid/hydrogen peroxide redox pair in order to improve COSs activity.

\section{MATERIALS AND METHODS}

\section{Materials}

Ascorbic acid (VWR, Leuven, Belgium), lactic acid (HiMedia, Mumbai, India), sodium bicarbonate (HiMedia, Mumbai, India), ethanol 99.99\% (HiMedia, Mumbai, India), sodium hydroxyl (Merck, Darmstadt, Germany). Syringic acid (HiMedia, Mumbai, India), hydrogen peroxide (HiMedia, Mumbai, India), nutrient broth (HiMedia, Mumbai, India), nutrient agar (HiMedia, Mumbai, India), folin (Merck, Darmstadt, Germany). TLC silica gel 60 F254 (Merck, Darmstadt, Germany). All other chemicals were of analytical grade, or of the highest grade, available commercially.

We used the COSs powder, left from our previous research. Briefly, $0.8 \%$ chitosan solution was prepared in $0.8 \%$ lactic acid solution. The chitosan solution was hydrolyzed by cellulase with optimal parameters. After hydrolysis, the COSs were filtered through an ultrafiltration membrane, with membranes ranging in molecular weight cut-offs (MWCO). COSs fractionation was carried out by spray drying (SD-06AG, LabPlant, UK), in order to created COSs powder. The COSs was packed in vacuum for storage ${ }^{19}$.

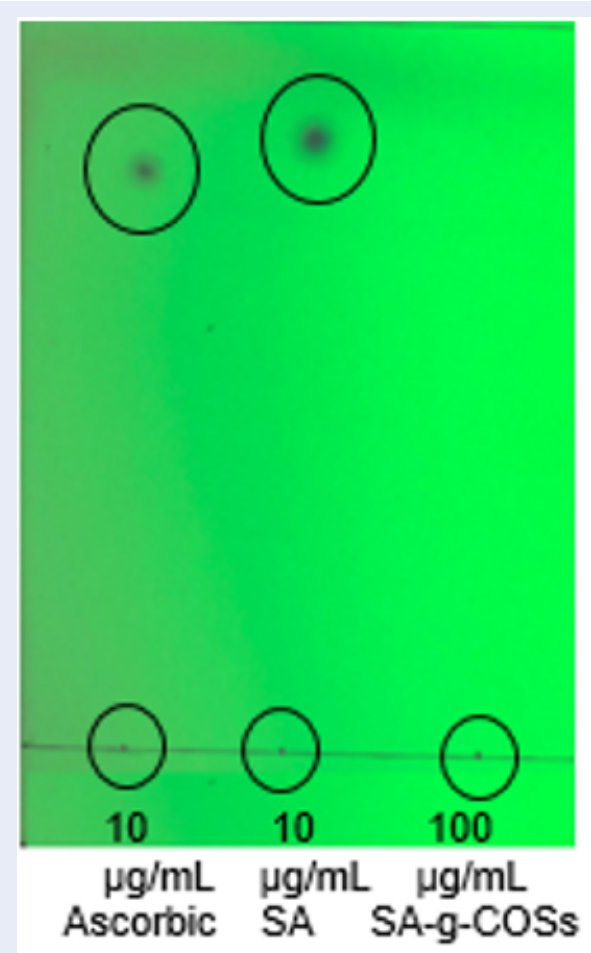

Figure 3: The TLC plate diagram of Ascorbic acid, syringic acid (SA) and SA-g-COSs. Syringic acid, ascorbic acid, and SA-g-COSs were developed on a silica gelplate () with chloroform - ethyl acetate - acetic acid (50:50:1) asmobile phase, heating at $100^{\circ} \mathrm{C}$ for $5 \mathrm{~min}$. The developed TLC plate was observed under UV light. The experiment repeated three times.

\section{Methods}

\section{Synthesis of syringic acid grafted chi- tooligosaccharides (SA-g-COSs)}

The synthesis of SA-g-COSs was performed according to the method of Curcio et al., (2009) with slight modifications ${ }^{18}$. Briefly, the COSs $(0.5 \mathrm{~g})$ was dissolved in $25 \mathrm{~mL}$ of water and then $1 \mathrm{~mL}$ of $1.0 \mathrm{M} \mathrm{H}_{2} \mathrm{O}_{2}$, containing $0.054 \mathrm{~g}$ of ascorbic acid was added. After $30 \mathrm{~min}$, the syringic acid was added to the mixture at different amounts, with the mass ratios of SA, conjugated onto COSs chain being 1:0.25, 1:0.5, 1:1 and 1:2. The $\mathrm{pH}$ value of mixture changed from 3 to 6 and the reaction was carried out at the temperature ranging from $27^{\circ} \mathrm{C}$ (room temperature) to $45^{\circ} \mathrm{C}$, for the periods of time, ranging from 2 to 24 hours. After the reaction, the mixture was allowed to rest at room temperature under atmospheric air, and then centrifuged with filter membrane $1 \mathrm{kDa}$ in $50 \mathrm{~mL}$ centrifuge tube, in order to remove unreacted syringic acid and the catalyst. Finally, the resulting solution was lyophilized, using 


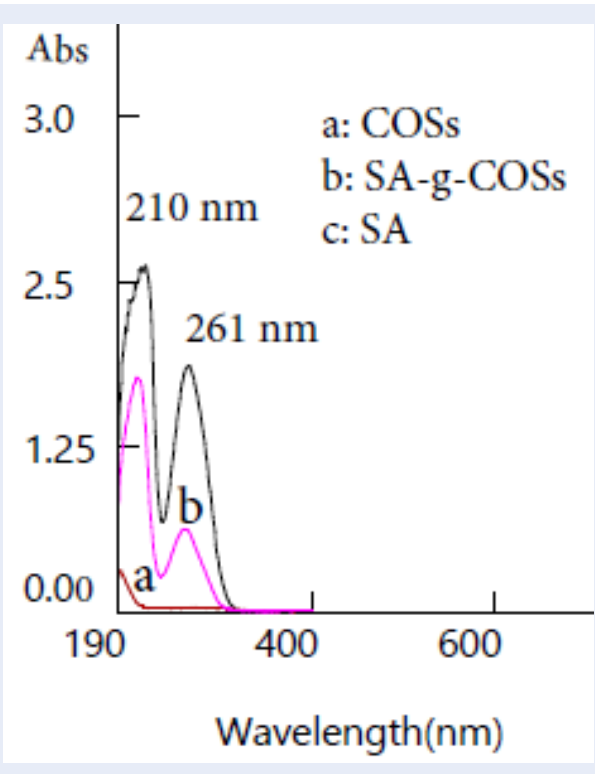

Figure 4: The UV-vis spectra of COSs (a), SA-gCOSs (b) and Syringic acid (c). The maximum absorbance of derivatives was determined by recording UV-vis absorption spectra (T60U UV-vis spectrophotometer, PG Instruments Ltd., US). The spectra of samples recorded at concentration of $50 \mathrm{ppm}$. The experiment repeated three times.

freeze dry system (FDU 2110, Eyela, Japan) to obtain SA-g-COSs solid samples (the moisture content of the SA-g-COSs, COSs and SA is the same).

\section{Characterization of SA-g-COSs}

To verify whether syringic acid was successfully grafted onto COSs backbones, TLC analysis was performed. Syringic acid, ascorbic acid, and SA-g-COSs were developed on a silica gel plate (TLC silica gel 60 F254, Merck, Darmstadt, Germany) with chloroform - ethyl acetate - acetic acid (50:50:1) as mobile phase, heating at $100^{\circ} \mathrm{C}$ for $5 \mathrm{~min}$. The developed TLC plate was observed under UV light; The SA-g-COSs was characterized by UV - vis, FT-IR. The maximum absorbance of derivatives was determined by recording UV-vis absorption spectra (T60U UV-vis spectrophotometer, PG Instruments Ltd., US). Original and conjugated COSs samples (25 to $100 \mathrm{ppm}$ ) were dissolved in deionized water and then UV-vis absorption spectra were recorded in full scan mode from 190 to $400 \mathrm{~nm}$. The FTIR spectra were recorded via the Alpha II FTIR spectrometer (Bruker, Germany). The dried sample was ground with potassium bromide $(\mathrm{KBr})$ powder and pressed into a pellet for spectrometric measurement in the frequency range of 4000 $400 \mathrm{~cm}-1^{20}$.

\section{Determination of SA content in SA-g-COSs}

The total syringic acid content of SA-g-COSs derivative was measured by the Folin - Ciocalteu method, describ ed by Liu, et al., (2013) with slight modifications ${ }^{16}$. Briefly, $1 \mathrm{~mL}$ of the sample solution was mixed with $1 \mathrm{~mL}$ of Folin-Ciocalteu reagent (10-fold dilution) and allowed to react at room temperature $\left(27 \pm 2^{\circ} \mathrm{C}\right)$ for $5 \mathrm{~min}$ in the dark. Then, $3 \mathrm{~mL}$ of $2 \% \mathrm{Na}_{2}$ $\mathrm{CO}_{3}$ was added and the mixture was allowed to stand for $30 \mathrm{~min}$ before measuring at A760 nm. The syringic acid was used as the standard and the results were expressed as mg of syringic acid per gram of SA-g-COSs dry weight (mg SA/g SA-g-COSs). The grafting degree was calculated from the SA content in the SA-gCOSs and the utilized amount of SA in the preparation of the SA-g-COSs.

\section{Antibacterial activity assay}

The antibacterial activity of derivatives was performed using agar disk diffusion test, then minimum inhibitory concentration (MIC) values were determined $^{21}$. Briefly, Indicator bacteria activated on nutrient broth medium (NB) $(10 \mathrm{~mL})$, The growth density was adjusted to match a MacFarland 0.5 standard $\left(10^{8} \mathrm{CFU} / \mathrm{mL}\right)$. A 1:100 diluted solution was prepared in a fresh NB and used as the inoculum $\left(10^{6}\right.$ $\mathrm{CFU} / \mathrm{mL})$. Each bacterial suspension $(100 \mu \mathrm{L})$ was uniformly spread on a nutrient agar medium disk. The five wells were formed on the surface of the agar disk $^{22} .100 \mu \mathrm{L}$ of the sample solution was poured to each well and plates were incubated for $18 \mathrm{~h}$ ours at $37^{\circ} \mathrm{C}$.

\section{Statistical Analysis}

Data were expressed as the mean \pm standard deviation (SD) of triplicates. The least significant difference (LSD), Duncan's multiple range test and one-way analysis of variance (ANOVA) were used for multiple comparisons by stratigraphic centurion. The difference was statistically significant if $\mathrm{p}<0.05$.

\section{RESULTS}

\section{Synthesis of syringic acid grafted chi- tooligosaccharides (SA-g-COSs)}

The principle of synthesis of SA-g-COSs states, that ascorbic acid solution is present as a di-acid and can further react with $\mathrm{H}_{2} \mathrm{O}_{2}$ to generate hydroxyl radical (HO •), as well as resonance, stabilized tricarbonyl ascorbate free radical $(\mathrm{AscH} \bullet)$. As $\mathrm{AscH} \bullet$ has a $\mathrm{pK}=-0.86$, it is not protonated and is present in the form of semi dehydroascorbate radical (Asc $\bullet-$ ). Then the previously formed $\mathrm{HO} \bullet$ attracts hydrogen 
from amino and hydroxyl group of COSs, resulting in COSs macro radicals. Finally, the corresponding syringic acid monomers, which are in the close vicinity of reaction sites become acceptors of COSs macro radicals. Thus, forming COSs-syringic acid conjugates (Figure 1) ${ }^{16}$.

The synthesis of derivative is influenced by factors, such as mass ratio of $\mathrm{SA}$ and COSs, $\mathrm{pH}$, temperature and time. Therefore, these factors were studied by single factor experiments. As shown in Figure 2, the SA contents in the SA-g-COSs increased with increasing mass ratio of SA to COSs chain as 1:0.25, $1: 0.5,1: 1,1: 2$ were $84.00,142.38,228.24,354.91 \mathrm{mg}$ SA/g SA-g-COSs, respectively. The grafting degrees of SA onto COSs were $18,91 \%, 30.75 \%, 26.42 \%$, and $21.42 \%$ with regard to $1: 0.25,1: 0.5,1: 1,1: 2$, respectively. According to our results, the optimal condition for the synthesis of SA-g-COSs was 1:0.5 as a mass ratio of SA to COSs chain. Because of different $\mathrm{pH}$ values in the synthesis process, the SA content in derivative is also different. The SA content in derivative showed an increase in $\mathrm{pH}$ values, ranged from 3 to 5 . The SA contents at $\mathrm{pH}$ of $3,4,5,6$ were $71.47,142.38,182.18,40.46 \mathrm{mg} \mathrm{SA} / \mathrm{g} \mathrm{SA}$-g-COSs, respectively. These results show, that the optimal condition for the synthesis of SA-g-COSs are when $\mathrm{pH}$ value is 5 . Our data are consistent with ascorbic acid stability in aqueous solution at $\mathrm{pH}$ levels from 4 to $6^{23}$. The research results show that when temperature increases, the SA content tends to reduce as 183.19 , $167.03,155.11,143.60 \mathrm{mg} \mathrm{SA} / \mathrm{g} \mathrm{SA}-\mathrm{g}-\mathrm{COSs}$. This result suggests the most effective temperature of synthesis process be $27^{\circ} \mathrm{C}$. Regarding the time of synthesis, it was increased when in the time ranged from $2 \mathrm{~h}$ to $6 \mathrm{~h}$ and then not changed until 24 hours. Therefore, the synthesis process stopped at $6 \mathrm{~h}$ with the SA content of $204.40 \mathrm{mg} \mathrm{SA} / \mathrm{g} \mathrm{SA}$-g-COSs, corresponding to grafting degree at $32 \%$.

\section{Characterization of SA-g-COSs}

The TLC result (Figure 3 ) showed that no free syringic acid in derivative was observed. Yet free syringic acid and ascorbic acid appeared on the developed plate. This demonstrated, that syringic acid successful grafted onto the COSs and this was not a mixture condition between syringic acid and COSs. The UV-vis spectra of SA, COSs, and SA-COSs are presented in Figure 4. The absorbance peak of SA-COSs showed, that COSs were well conjugated with SA by the ascorbic acid/hydrogen peroxide catalyst. The COSs did not yield an absorbance peak in the range from 200 to $400 \mathrm{~nm}$, whereas SA-COSs had two absorbance peaks at 210 and $261 \mathrm{~nm}$. This pattern is identical to the characteristic absorbance peak of SA, which should be assigned to the $\pi$-system of the benzene ring ${ }^{24}$, The results indicate, that SA was successfully grafted onto COSs. In the FT-IR spectra, SACOS shows absorbance significant peaks in 1732 and $1632 \mathrm{~cm}^{-1}$, implying both ester and amide stretching, respectively $^{25}$. Thus, our FTIR spectra in Figure 5indicate, that syringic acid was successfully conjugated onto COSs.

\section{Antibacterial activity}

The MIC values of COSs and SA-COSs derivative were determined as an evaluation of their antimicrobial activity against selected foodborne pathogenic bacteria. The result presented in Table 1 shows the minimum inhibitory concentration of COSs derivative against the growth of Escherichia coli and Bacillus subtilis. The COSs did not inhibit growth for both bacteria species at concentrations, ranged from 0.5 to $4 \%$. Yet, the derivative had a positive result for both bacteria species at $0.5 \%$, but negative result for both bacteria species at higher concentrations, ranged from 1 to $4 \%$. Therefore, the MIC of the derivative was determined at $1 \%$ concentration. This result shows that SA grafted on COSs chain increased antibacterial activity of COSs.

\section{DISCUSSION}

The results of this study show, that with the appropriate parameters, such as certain mass ratio between syringic acid and COSs $(0.5: 1), \mathrm{pH} 5$, temperature $\left(27^{\circ} \mathrm{C}\right)$, time (6 hours), the obtained derivative has syringic content, equal to $204 \mathrm{mg} / \mathrm{g}$, corresponding to the level of grafting equal to $32 \%$. The results of this study are similar to previous study, if mass ratio of gallic acid to chitosan is $0.5: 1^{24}$. The enhancement of grafting degree was possibly due to the increasing of SA mass ratio within the critical range. However, beyond that range, the excess of free SA molecules may inhibit grafting process, causing a reduction of grafting degree ${ }^{16}$. Our results offer the better grafting degree than the previous study of Eom et al., (2012) when N,N'-dicyclohexylcarbodiimide catalyst was used to conjugate phenolic acid onto COSs with the grafting degree at $10.26 \%$. However, in that work, the factors which affected the synthesis process were not presented ${ }^{14}$. Liu et al., (2013) reported the synthesis of chitosan-gallic acid conjugate. The result of which shows gallic acid content at $128.3 \mathrm{mg} / \mathrm{g}$ chitosan-gallic acid, however, the study did not investigate the optimal conditions, such as $\mathrm{pH}$, temperature and time of synthesis ${ }^{16}$. Cho et al., (2011) showed, that the synthesis of gallic acid grafted chitosan had the highest grafting degree at $53.88 \%{ }^{20}$. 

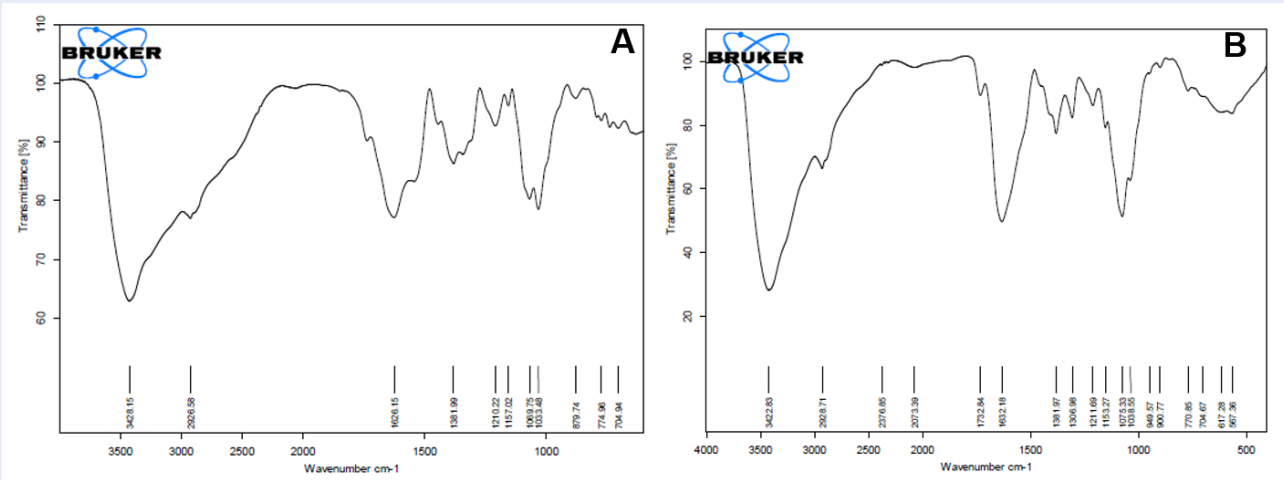

Figure 5: FT-IR spectra of COSs (A) and SA-g-COSs (B). The FT-IR spectra were recorded in powder form in $\mathrm{KBr}$ discs in the range of $4000-400 \mathrm{~cm}^{-1}$ (Brucker, Germany). The experiment repeated three times.
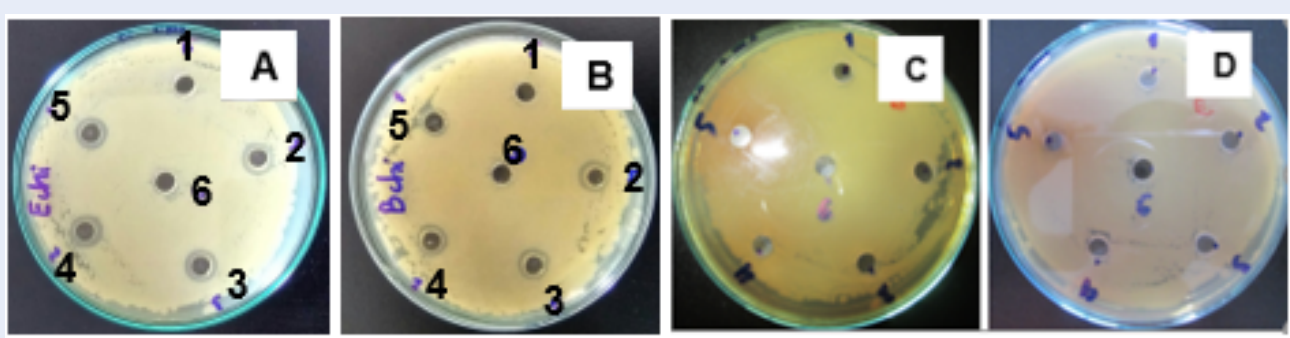

Figure 6: Antibacterial activity of COSs and derivatives by agar disk diffusion test. Concentrations ranged from 0.5 to $4 \%$, (corresponding number changed from 1 to 5 , control sample at centre) for each plate. A and $B$ figures show result of derivatives against growth of Escherichia coli and Bacillus subtilis, respectively. C and D figures show result of COSs not against growth of Escherichia coli and Bacillus subtilis, respectively.

Table 1: The MIC results of COSs and SA-g-COSs

\begin{tabular}{lccccccccccccc}
\hline Bacteria & \multicolumn{3}{c}{ Escherichia coli (G-) } & \multicolumn{7}{c}{ Bacillus subtilis (G+) } \\
$\begin{array}{l}\text { Concentration } \\
(\%)\end{array}$ & 0.5 & 1.0 & 2.0 & 3.0 & 4.0 & Control & 0.5 & 1.0 & 2.0 & 3.0 & 4.0 & Control \\
COSs & + & + & + & + & + & + & + & + & + & + & + & + \\
SA-g-COSs & + & - & - & - & - & + & + & - & - & - & - & +
\end{tabular}

$(+)$ not appearance of inhibition zone, (-) appearance of inhibition zone. The experiment repeated three times ( $n=3)$.

\section{CONCLUSION}

The catalyst of an ascorbic acid/hydrogen peroxide redox pair, which was used to graft SA onto COSs, was successfully achieved in inert atmosphere. The best conditions for the synthesis had the mass ratio between syringic acid and COSs at 0.5:1, $\mathrm{pH} 5$, temperature $27^{\circ} \mathrm{C}$, time - 6 hours. Antibacterial assays showed that SA, grafted onto COSs improved COSs activity, comparing to free COSs.

\section{ABBREVIATIONS}

CFU: Colony forming unit
COSs: Chitooligosaccharides

FT-IR: Fourier Transform Infrared

MIC: Minimum inhibitory concentration

SA: Syringic acid

TLC: Thin Layer Chromatography

UV-vis: Ultraviolet-Visible

\section{COMPETING INTERESTS}

The authors declare that they have no competing interest. 


\section{AUTHORS' CONTRIBUTIONS}

All authors of this manuscript have contributed to the work and approved contents of the final version.

\section{ACKNOWLEDGMENTS}

This study was supported by Vietnam National Foundation for Science and Technology Development (NAFOSTED) under Grant Number: 106.022019.47; the government's 911 research fund; inomarVNUHCM Institute; Biochemistry Lab, Faculty of Biology-Biotechnology, University of Science, VNUHCM and Ho Chi Minh City University of Food Industry (HUFI).

\section{REFERENCES}

1. Rinaudo M. Chitin and chitosan: properties and applications. Progress in polymer science. 2006;31(7):603-632

2. Ngo DN, et al. Production of chitin oligosaccharides with different molecular weights and their antioxidant effect in RAW 264.7 cells. Journal of Functional Foods. 2009;1(2):188-198.

3. Seferian PG, Martinez ML. Immune stimulating activity of two new chitosan containing adjuvant formulations. Vaccine. 2000;19(6):661-668.

4. Kim SK, Nghiep ND, Rajapakse N. Therapeutic prospectives of chitin, chitosan and their derivatives. J chitin chitosan. 2006;11(1):1-10.

5. Park PJ, Je JY, Kim SK. Free radical scavenging activities of differently deacetylated chitosans using an ESR spectrometer. Carbohydrate Polymers. 2004;55(1):17-22.

6. Zhao XR, Xia WS. Antimicrobial activities of chitosan and applications in food preservation. Food Research and Development. 2006;27(2):157-160.

7. Yang TC, Chou CC, Li CF. Antibacterial activity of N-alkylated disaccharide chitosan derivatives. International Journal of Food Microbiology. 2005;97(3):237-245

8. Chang $\mathrm{H}$, Chen J, Fang F. Enhancement of Antibody Response by Chitosan, A Novel Adj uvant of Inactivated Influenza Vaccine. Chinese Journal of Biologicals. 2004;17(6):383-385.

9. Huang $\mathrm{R}$, et al. Strong electronic charge as an important factor for anticancer activity of chitooligosaccharides (COS). Life Sciences. 2006;78(20):2399-2408.

10. Kumar MNR. A review of chitin and chitosan applications. Reactive and functional polymers. 2000;46(1):1-27.
11. Ngo DN, Kim MM, Kim SK. Chitin oligosaccharides inhibit oxidative stress in live cells. Carbohydrate Polymers. 2008;74(2):228-234

12. $\mathrm{Ngo} \mathrm{DH}$, et al. Gallyl chitooligosaccharides inhibit intracellular free radical-mediated oxidation. Food Chem;128(4):974-981.

13. Kim SK, Rajapakse N. Enzymatic production and biological activities of chitosan oligosaccharides (COS): A review. Carbohydrate polymers. 2005;62(4):357-368.

14. Eom TK, Senevirathne M, Kim SK. Synthesis of phenolic acid conjugated chitooligosaccharides and evaluation of their antioxidant activity. Environ Toxicol Pharmacol. 2012;34(2):519527.

15. Vo TS, Ngo DH, Kim SK. Gallic acid-grafted chitooligosaccharides suppress antigen-induced allergic reactions in $\mathrm{RBL}-2 \mathrm{H} 3$ mast cells. Eur J Pharm Sci;2(527-33).

16. Liu J, Lu JF, Kan J, Jin CH. Synthesis of chitosan-gallic acid conjugate: structure characterization and in vitro anti-diabetic potential. Int J Biol Macromol;62:321-9.

17. Martins $S$, et al. Bioactive phenolic compounds: production and extraction by solid-state fermentation. A review. Biotechnol Adv. 2011;29(3):365-73.

18. Curcio $\mathrm{M}$, et al. Covalent insertion of antioxidant molecules on chitosan by a free radical grafting procedure. J Agric Food Chem. 2009;57(13):5933-8.

19. Bui, et al. Research antioxidant activity of chitooligosacchride by uv-vis absorption spectrocopy. Journal of Science Ho Chi Minh City Open University. 2018;8(1):64-69.

20. Cho YS, et al. Preparation, characterization, and antioxidant properties of gallic acid-grafted-chitosans. Carbohydrate Polymers;83(4):1617-1622.

21. Ruparelia JP, et al. Strain specificity in antimicrobial activity of silver and copper nanoparticles. Acta Biomater. 2008;4(3):707-16.

22. Islam MA, et al. Determination of minimum inhibitory concentration (MIC) of cloxacillin for selected isolates of methicillin-resistant Staphylococcus aureus (MRSA) with their antibiogram. Bangladesh Journal of Veterinary Medicine. 2008;6(1):121-126.

23. Ahmad l, et al. Effect of stabilizers on the chemical and photodegradation of ascorbic acid in aqueous solution. Baqai Journal of Health Sciences. 2016;19(1):3-11.

24. $\mathrm{Hu} \mathrm{Q}$, et al. In Vitro Antioxidant-Activity Evaluation of Gallic-Acid-Grafted Chitosan Conjugate Synthesized by FreeRadical-Induced Grafting Method. J Agric Food Chem. 2016;64(29):5893-900.

25. Pasanphan W, Buettner GR, Chirachanchai S. Chitosan gallate as a novel potential polysaccharide antioxidant: an EPR study Carbohydr Res. 2010;345(1):132-40. 